\title{
Assessing decision-making capacity
}

\author{
Vera Prisacari, MD
}

$\mathrm{M}$ edical and surgical inpatient teams often consult psychiatrists to help assess a patient's decision-making capacity (DMC). Completing a DMC assessment can be a source of stress and frustration for psychiatry residents; however, the process can be significantly improved by following a consistent and focused psychiatric interview. In our institution, the most frequently used tool for assessing DMC is the Aid to Capacity Evaluation (ACE). ${ }^{1}$ The American Academy of Family Physicians offers a printer-friendly adaptation ${ }^{2}$ of the ACE assessment.

\section{Reasons for a DMC consultation}

When accepting a DMC consultation, make sure to specify for which medical decision the primary team would like the patient to be evaluated so that the consultation can be most helpful and specific to primary team's concerns. ${ }^{3}$ The 4 most common reasons for a DMC consultation are:

Acute changes in mental status. These changes may be due to hypoxia, infection, medication, metabolic disturbances, or other medical conditions. Often, the diagnosis is delirium due to a medical condition, and assessing for DMC is deferred until the delirium resolves.

Refusal of a recommended treatment. One of the most frequent reasons for a DMC consultation is when a patient declines the primary team's treatment recommendations. These recommendations may include medications, interventions

(procedural or surgical), or discharge planning (such as transfer to a rehabilitation facility or skilled nursing care facility).

Consenting to a risky or invasive treatment too hastily. This occurs less often than the other 3 scenarios, most likely because capacity is rarely questioned when a patient's decision aligns with the physicians' recommendations.

Having risk factors for impaired decision-making. One of the most common reasons for involving psychiatry in a DMC consultation is when the patient has a risk factor that may impair his/her decision-making.

These risk factors include:

- a chronic psychiatric or neurologic condition

- a significant cultural or language barrier

- a low or unknown education level

- anxiety or discomfort with institutional health care settings

- age $<18$ or $>85$.

\section{When should you put off a DMC consultation?}

There are situations in which a DMC consultation should be declined or postponed.

continued

LET YOUR VOICE BE HEARD

CURRENT PSYCHIATRY invites psychiatry residents to share their views on professional or clinical topics for publication in Residents' Voices. E-mail jbauer@mdedge.com for author guidelines.

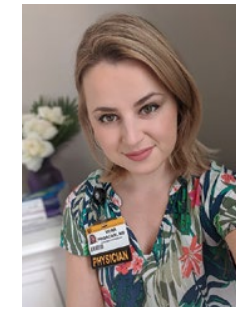

Dr. Prisacari is a PGY-3 Psychiatry Resident, Department of Psychiatry, University of Missouri School of Medicine, Columbia, Missouri.

\section{Disclosure}

The author reports no financial relationships with any companies whose products are mentioned in this article, or with manufacturers of competing products.

doi: $10.12788 /$ cp.0026 


\section{Clinical Point}

\section{Because patients are presumed to be capable, if the determination is not clear, err on the side of capacity}

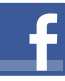

Discuss this article at www.facebook.com/ MDedgePsychiatry
A DMC consultation is not appropriate for patients who have a court-appointed legal guardian because these patients are considered legally incapacitated. Although such patients may still choose to participate in shared decision-making with the treatment team and communicate those decisions to their legal guardian, all treatment decisions are made by their legal guardian.

Consider delaying DMC consultations for patients with acute changes in mental status, including delirium, or those who are sedated or intubated. On the other hand, patients not excluded from a DMC assessment include those with mild or major neurocognitive disorders (such as Alzheimer's disease or other types of dementia), intellectual disabilities, a durable power of attorney (DPOA), or legal charges. It is important to note that a diagnosis of a neurocognitive disorder does not preclude capacity, and capacity may therefore vary based on the complexity of medical decision.

\section{Prepare for the interview}

Prior to meeting the patient, familiarize yourself with his/her reason for admission, diagnosis, medical workup, treatment provided thus far, and proposed future treatment. If possible, attempt to gather the patient's medical and psychiatric history from chart review, which may be obtained from outside medical records or inferred from the patient's current medication list. Review the chart for any signs that the patient is in a delirious state, including the use of psychotropic medications for agitation, restraints, or a sitter at bedside. Finally, speak with the patient's nurse before entering the patient's room. This may provide valuable information regarding the patient's willingness to participate (including any hostility or aggression toward clinicians), any barriers to the interview (such as hearing or language difficulties), and if any family or friends are present at the bedside.

\section{During the interview}

The structure of a decision-making capacity evaluation has been well documented elsewhere $^{1,2}$ and, if completed, is sufficient for determining DMC. Asking the patient about additional psychiatric histories, including hospitalizations, suicide attempts, or a family history of psychiatric illness, may be counterproductive and frustrating for the patient, especially if the interview has already been lengthy and emotionally exhausting. Therefore, it is often appropriate to shorten the psychiatric interview to assess for symptoms of anxiety and depression, perform a brief suicide risk assessment, and assess for the presence of auditory or visual hallucinations, or delusions. This allows for a complete mental status exam while still focusing the interview on determining DMC. If time allows and the patient is willing to participate, it can be helpful to perform a Montreal Cognitive Assessment (MoCA) or Mini-Mental State Exam, although a score that indicates some level of cognitive impairment $\left(<26\right.$ on $\left.\mathrm{MoCA}^{4}\right)$ does not necessarily preclude patient from having capacity for certain medical decisions. Additionally, if the patient does not understand a question, explain it and return to it later to check for comprehension.

\section{Making a recommendation}

Patients are presumed to be capable; therefore, if the determination is not clear, err on the side of capacity. Additional information may be helpful from the patient's family, friends, DPOA, or guardian, especially as it pertains to the patient's past wishes and medical decisions made in similar situations. If available and pertinent, review the patient's advance health care directive.

Communicate your recommendations to the primary team clearly and concisely, including if the evaluation is incomplete, and if the patient will be seen again by the consult team, because this may determine 
disposition planning. Finally, if the patient is deemed to not have DMC, the primary team must then establish a surrogate decision maker on the patient's behalf. Because the protocol and hierarchy of next-of-kin varies by state law and institution policy, it is essential to involve social work and case management.

\section{References}

1. Joint Centre for Bioethics. Aid to capacity evaluation (ACE) http://www.jcb.utoronto.ca/tools/documents/ace.pdf. Published 1996. Accessed July 20, 2020.

2. American Academy of Family Physicians. Aid to capacity evaluation. https://www.aafp.org/afp/2001/0715/afp 20010715p299-f2.pdf. Published 2000. Accessed July 20, 2020.

3. Tunzi M. Can the patient decide? Evaluating patient capacity in practice. Am Fam Physician. 2001;64(2): 299-306.

4. Montreal Cognitive Assessment. FAQ. https://www. mocatest.org/faq/. Accessed July 20, 2020.

\section{Clinical Point}

If the patient is deemed to not have DMC, the primary team must establish a surrogate decision maker on the patient's behalf 